\title{
General Analytical Procedure for Determination of Acidity Parameters of Weak Acids and Bases
}

\author{
Bogusław Pilarski, ${ }^{1}$ Roman Kaliszan, ${ }^{2}$ Dariusz Wyrzykowski, ${ }^{3}$ \\ Janusz Młodzianowski, ${ }^{1}$ and Agata Balińska ${ }^{1,2}$ \\ ${ }^{1}$ Cerko Sp. z o.o. Sp.K, Al. Zwycięstwa 96/98, 81-451 Gdynia, Poland \\ ${ }^{2}$ Department of Biopharmaceutics and Pharmacodynamics, Medical University of Gdańsk, Gen. J. Hallera 107, 80-416 Gdańsk, Poland \\ ${ }^{3}$ Faculty of Chemistry, University of Gdańsk, Wita Stwosza 63, 80-308 Gdańsk, Poland \\ Correspondence should be addressed to Bogusław Pilarski; bpilarski@cerko.pl
}

Received 8 October 2014; Accepted 2 January 2015

Academic Editor: Fábio Rodrigo Piovezan Rocha

Copyright (C) 2015 Bogusław Pilarski et al. This is an open access article distributed under the Creative Commons Attribution License, which permits unrestricted use, distribution, and reproduction in any medium, provided the original work is properly cited.

The paper presents a new convenient, inexpensive, and reagent-saving general methodology for the determination of $\mathrm{p} K_{\mathrm{a}}$ values for components of the mixture of diverse chemical classes weak organic acids and bases in water solution, without the need to separate individual analytes. The data obtained from simple $\mathrm{pH}$-metric microtitrations are numerically processed into reliable $\mathrm{p} K_{\mathrm{a}}$ values for each component of the mixture. Excellent agreement has been obtained between the determined $p K_{\mathrm{a}}$ values and the reference literature data for compounds studied.

\section{Introduction}

Prediction or determination of $\mathrm{p} K_{\mathrm{a}}$ value is of great importance in chemistry, in particular in life and material sciences, pharmaceutical industry, and other R\&D oriented enterprises. Important drug properties, such as lipophilicity, solubility, and transmembrane transfer, are all $\mathrm{pH}$ dependent. Also, rational drug formulation requires the knowledge of $\mathrm{p} K_{\mathrm{a}}$. The proportion of drugs with an ionizable group has been estimated at $95 \%$ [1], but only $62.9 \%$ of drugs under analysis were ionizable at $\mathrm{pH} 2-12$ [2]. According to Wells data $75 \%$ of drugs are weak bases and $20 \%$ weak acids and the remaining contain nonionics, ampholytes, and alcohols [1].

Recently, some theoretical approaches were employed to predict the $\mathrm{p} K_{\mathrm{a}}$ value, for example, ab initio quantum mechanical calculations $[3,4]$ or QSPR (quantitative structure-property relationship) modeling $[5,6]$ as well as QSPR models which employ partial atomic charges as descriptors $[7,8]$. The theoretical models take into account electronic effects (induction, resonance), solvation of compounds of type $\mathrm{HA}, \mathrm{BH}$ and their ionic forms, that is,
$\mathrm{A}^{-}$and $\mathrm{BH}^{+}$, hydrogen bonding, and various stereochemical effects.

This report presents an application of $\mathrm{pH}$-metric microtitration to determine standard $\mathrm{p} K_{\mathrm{a}}$ parameters of components of mixtures of various weak acids and bases by employing a technologically advanced potentiometer device and a software based on an algorithm straightforwardly accounting for complex acid-base equilibria (see below). A composition of the mixtures under study can be expressed as follows:

$$
\begin{aligned}
\mathrm{H}_{3} \mathrm{~A}_{1} & +\mathrm{H}_{2} \mathrm{~A}_{2}+\mathrm{HA}_{3}+\mathrm{B}_{1}+\mathrm{B}_{2}+\left(\mathrm{R}_{1} \mathrm{R}_{2}\right) \mathrm{CH}_{2} \\
+ & \left(\mathrm{R}_{1} \mathrm{R}_{2}\right) \mathrm{N}-\mathrm{H}+\mathrm{Ar}-\mathrm{OH}
\end{aligned}
$$

where $\mathrm{H}_{3} \mathrm{~A}_{1}+\mathrm{H}_{2} \mathrm{~A}_{2}+\mathrm{HA}_{3}$ represents 3-H, 2-H, and 1-H protic carboxylic acids, $\mathrm{B}_{1}+\mathrm{B}_{2}$ represents organic bases, $\left(\mathrm{R}_{1} \mathrm{R}_{2}\right) \mathrm{CH}_{2}$ represents the so-called $\mathrm{C}-\mathrm{H}$ acids, $\mathrm{R}_{1} \mathrm{R}_{2} \mathrm{~N}-$ $\mathrm{H}$ - represents the $\mathrm{N}-\mathrm{H}$ acids, and $\mathrm{ArOH}$ denotes phenolic/enolic moiety $(\mathrm{O}-\mathrm{H}$ acids). The $\mathrm{C}-\mathrm{H}, \mathrm{N}-\mathrm{H}$, and $\mathrm{O}-\mathrm{H}$ acids are often reported as tautomeric forms of heterocyclic compounds with pharmacological activity and are identified 
TABLE 1: Stoichiometric matrix for model 1.

\begin{tabular}{lccccc}
\hline & $\mathrm{H}^{+1}$ & $\mathrm{H}_{2} \mathrm{~A}$ & $\mathrm{HA}^{-1}$ & $\mathrm{~A}^{-2}$ & $\mathrm{OH}^{-1}$ \\
\hline $\mathrm{p} K_{1}$ & 1 & -1 & 1 & 0 & 0 \\
$\mathrm{p} K_{2}$ & 1 & 0 & -1 & 1 & 0 \\
$\mathrm{p} K_{\mathrm{w}}$ & 1 & 0 & 0 & 0 & 1 \\
\hline
\end{tabular}

0 denotes a species that does not take part in equilibrium; -1 donates substrate (left side of equilibria equation); 1 donates product (right side of equilibria equation).

within different groups of natural compounds (flavonoids, quinines, etc.).

Numerical Modelling. Numerical procedures are based on an original algorithm elaborated by Kostrowicki and Liwo [9] as well as the CVEQUID program, which was adopted in the Cerko Lab software within the Cerko Lab System microtitrator unit (Cerko, Gdynia, Poland). All details concerning Kostrowicki and Liwo algorithm were described previously [10]. The CVEQUID program is based on a least-square method for the determination of all parameters and takes into account all the sources of experimental errors considered in potentiometry, that is,

(a) electrode calibration parameters $\left(E^{0}\right.$, the standard potential (cell constant) and $S$, the standard Nernstian slope parameter);

(b) composition of titrand $\mathrm{D}$, its concentration $C_{0}$ $(\mathrm{mol} / \mathrm{L})$, and volume $V_{0}(\mathrm{~mL})$;

(c) composition of titrant $\mathrm{T}$, its concentration $(\mathrm{mol} / \mathrm{L})$, and added volume $(\mathrm{mL})$;

(d) measured EMF (the electromotive force) in $\mathrm{mV}$.

Within the Cerko Lab System software, the equilibrium is denoted as model. The model consists of a set of equations. Each equation is related to a particular $\mathrm{p} K$ value and to $\mathrm{p} K_{\mathrm{w}}$. The model includes also information about the composition of titrant $\mathrm{T}$ and titrand $\mathrm{D}$. The stoichiometric matrix, required for the numerical procedures, is generated from the model automatically. The representative models and the corresponding stoichiometric matrix for $\mathrm{H}_{2} \mathrm{~A}$ (model 1) as well as $\mathrm{H}_{3} \mathrm{~A}+$ $\mathrm{H}_{2} \mathrm{~A}_{1}+\mathrm{HA}_{2}$ (model 2) systems are given below.

Model 1. Reagents include titrand $\mathrm{D}=\mathrm{H}_{2} \mathrm{~A}$ and titrant $\mathrm{T}=\mathrm{OH}$. Individual equilibria that contribute to the overall equilibrium of the system are as follows:

(1) $\mathrm{H}_{2} \mathrm{~A}=\mathrm{H}^{+1}+\mathrm{HA}^{-1}, K_{1}=\left[\mathrm{H}^{+1}\right]\left[\mathrm{HA}^{-1}\right] /\left[\mathrm{H}_{2} \mathrm{~A}\right]$,

(2) $\mathrm{HA}^{-1}=\mathrm{H}^{+}+\mathrm{A}^{-2}, K_{2}=\left[\mathrm{H}^{+1}\right]\left[\mathrm{A}^{-2}\right] /\left[\mathrm{HA}^{-1}\right]$,

(3) $0=\mathrm{H}^{+1}+\mathrm{OH}^{-1}, K_{\mathrm{w}}=K\left[\mathrm{H}_{2} \mathrm{O}\right]=\left[\mathrm{H}^{+1}\right]\left[\mathrm{OH}^{-1}\right]$.

The stoichiometric matrix for the above model is presented in Table 1 .

The concentration of titrand, $\mathrm{D}\left(C_{0}=C_{01}+C_{02}+C_{03} \cdots\right)$, and titrand volume $\left(V_{0}=V_{01}+V_{02}+V_{03} \cdots\right)$, used in the potentiometric titration, result from mixing of various types of acid-base solutions.

Model 2. Reagents include titrand $\mathrm{D}=\mathrm{H}_{3} \mathrm{~A}+\mathrm{H}_{2} \mathrm{~A}_{1}+\mathrm{HA}_{2}$ and titrant $\mathrm{T}=\mathrm{OH}$ (Table 2). The model consists of a water

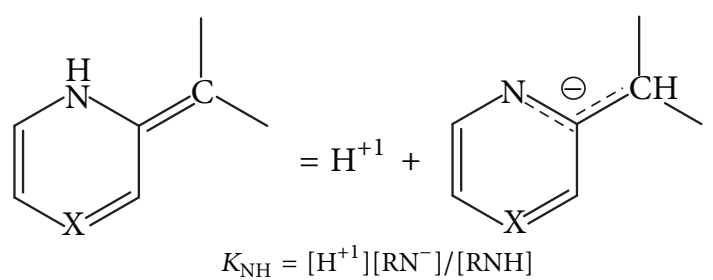

SCHEME 1

solution of different types of acids. The equilibrium constants and stoichiometric matrix are as follows:

(1) $\mathrm{H}_{3} \mathrm{~A}=\mathrm{H}^{+1}+\mathrm{H}_{2} \mathrm{~A}^{-1}, K_{1 \mathrm{~A}}=\left[\mathrm{H}^{+1}\right]\left[\mathrm{H}_{2} \mathrm{~A}^{-1}\right] /\left[\mathrm{H}_{3} \mathrm{~A}\right]$,

(2) $\mathrm{H}_{2} \mathrm{~A}^{-1}=\mathrm{H}^{+1}+\mathrm{HA}^{-2}, K_{2 \mathrm{~A}}=\left[\mathrm{H}^{+1}\right]\left[\mathrm{HA}^{-2}\right] /\left[\mathrm{H}_{2} \mathrm{~A}^{-1}\right]$,

(3) $\mathrm{HA}^{-2}=\mathrm{H}^{+}+\mathrm{A}^{-3}, K_{3 \mathrm{~A}}=\left[\mathrm{H}^{+1}\right]\left[\mathrm{A}^{-3}\right] /\left[\mathrm{HA}^{-2}\right]$,

(4) $\mathrm{H}_{2} \mathrm{~A}_{1}=\mathrm{H}^{+1}+\mathrm{HA}_{1}^{-1}, K_{1 \mathrm{~A}_{1}}=\left[\mathrm{H}^{+1}\right]\left[\mathrm{HA}_{1}^{-1}\right] /\left[\mathrm{H}_{2} \mathrm{~A}_{1}\right]$,

(5) $\mathrm{HA}_{1}^{-1}=\mathrm{H}^{+}+\mathrm{A}_{1}^{-2}, K_{2 \mathrm{~A}_{1}}=\left[\mathrm{H}^{+1}\right]\left[\mathrm{A}_{1}^{-2}\right] /\left[\mathrm{HA}_{1}{ }^{-1}\right]$,

(6) $\mathrm{HA}_{2}=\mathrm{H}^{+1}+\mathrm{A}_{2}^{-1}, K_{1 \mathrm{~A}_{2}}=\left[\mathrm{H}^{+1}\right]\left[\mathrm{A}_{2}^{-1}\right] /\left[\mathrm{HA}_{2}\right]$,

(7) $0=\mathrm{H}^{+1}+\mathrm{OH}^{-1}, K_{\mathrm{w}}=K\left[\mathrm{H}_{2} \mathrm{O}\right]=\left[\mathrm{H}^{+1}\right]\left[\mathrm{OH}^{-1}\right]$.

General Model. Reagents include titrand $\mathrm{D}=\mathrm{H}_{3} \mathrm{~A}+$ $\mathrm{H}_{2} \mathrm{~A}_{1}+\mathrm{HA}_{2}+\mathrm{B}+\mathrm{B}_{1}+\left(\mathrm{R}_{1} \mathrm{R}_{2}\right) \mathrm{CH}_{2}+\mathrm{R}_{1} \mathrm{R}_{2} \mathrm{NH}+$ $\mathrm{Ar}-\mathrm{OH}+n \mathrm{HX}$ (HX as a strong mineral acid) ..., and titrant $\mathrm{T}=\mathrm{OH}$. The presence of a strong mineral acid causes transformation of all the basic reagents into an acidic form-conjugate acid of amine.

The model consists of a water solution of different types of acids, bases, $\mathrm{C}-\mathrm{H}, \mathrm{N}-\mathrm{H}$, and $\mathrm{O}-\mathrm{H}$ acids. The existing equilibria in solution are given below:

(1) $\mathrm{H}_{3} \mathrm{~A}=\mathrm{H}^{+1}+\mathrm{H}_{2} \mathrm{~A}^{-1}, K_{1 \mathrm{~A}}=\left[\mathrm{H}^{+1}\right]\left[\mathrm{H}_{2} \mathrm{~A}^{-1}\right] /\left[\mathrm{H}_{3} \mathrm{~A}\right]$,

(2) $\mathrm{H}_{2} \mathrm{~A}^{-1}=\mathrm{H}^{+1}+\mathrm{HA}^{-2}, K_{2 \mathrm{~A}}=\left[\mathrm{H}^{+1}\right]\left[\mathrm{HA}^{-2}\right] /\left[\mathrm{H}_{2} \mathrm{~A}^{-1}\right]$,

(3) $\mathrm{HA}^{-2}=\mathrm{H}^{+}+\mathrm{A}^{-3}, K_{3 \mathrm{~A}}=\left[\mathrm{H}^{+1}\right]\left[\mathrm{A}^{-3}\right] /\left[\mathrm{HA}^{-2}\right]$,

(4) $\mathrm{H}_{2} \mathrm{~A}_{1}=\mathrm{H}^{+1}+\mathrm{HA}_{1}^{-1}, K_{1 \mathrm{~A}_{1}}=\left[\mathrm{H}^{+1}\right]\left[\mathrm{HA}_{1}^{-1}\right] /\left[\mathrm{H}_{2} \mathrm{~A}_{1}\right]$,

(5) $\mathrm{HA}_{1}^{-1}=\mathrm{H}^{+}+\mathrm{A}_{1}^{-2}, K_{2 \mathrm{~A}_{1}}=\left[\mathrm{H}^{+1}\right]\left[\mathrm{A}_{1}^{-2}\right] /\left[\mathrm{HA}_{1}{ }^{-1}\right]$,

(6) $\mathrm{HA}_{2}=\mathrm{H}^{+1}+\mathrm{A}_{2}^{-1}, K_{1 \mathrm{~A}_{2}}=\left[\mathrm{H}^{+1}\right]\left[\mathrm{A}_{2}^{-1}\right] /\left[\mathrm{HA}_{2}\right]$,

(7) $\mathrm{BH}^{+1}=\mathrm{H}^{+1}+\mathrm{B}, K_{\mathrm{BH}^{+1}}=\left[\mathrm{H}^{+1}\right][\mathrm{B}] /\left[\mathrm{BH}^{+1}\right]$,

(8) $\left(\mathrm{R}_{1} \mathrm{R}_{2}\right) \mathrm{CH}_{2}=\mathrm{H}^{+1}+\mathrm{R}_{1} \mathrm{R}_{2} \mathrm{CH}^{-1}, K_{\left(\mathrm{R}_{1} \mathrm{R}_{2}\right) \mathrm{CH}_{2}}=\left[\mathrm{H}^{+1}\right]$ $\left[\left(\mathrm{R}_{1} \mathrm{R}_{2}\right) \mathrm{CH}^{-1}\right] /\left[\left(\mathrm{R}_{1} \mathrm{R}_{2}\right) \mathrm{CH}_{2}\right]$,

(9) see Scheme 1,

(10) $\mathrm{ArOH}=\mathrm{H}^{+1}+\mathrm{ArO}^{-1}, K_{\mathrm{OH}}=\left[\mathrm{H}^{+1}\right]\left[\mathrm{ArO}^{-1}\right] /$ $[\mathrm{ArOH}]$,

(11) $\mathrm{H}_{2} \mathrm{O}=\mathrm{H}^{+1}+\mathrm{OH}^{-1}, K_{\mathrm{w}}=K\left[\mathrm{H}_{2} \mathrm{O}\right]=\left[\mathrm{H}^{+1}\right]\left[\mathrm{OH}^{-1}\right]$.

\section{Experimental}

2.1. Apparatus and Reagents. The $\mathrm{pH}$-metric titrations were performed in a $30 \mathrm{~mL}$ thermostated $\left(25.0 \pm 0.2^{\circ} \mathrm{C}\right)$ cell, using a Cerko Lab microtitration unit, fitted with a $\mathrm{pH}$ electrode (Hydromet ERH-13-6). The temperature was controlled using 
TABLE 2: Stoichiometric matrix for model 2.

\begin{tabular}{lcccccccccccc}
\hline & $\mathrm{H}^{+1}$ & $\mathrm{H}_{3} \mathrm{~A}$ & $\mathrm{H}_{2} \mathrm{~A}^{-1}$ & $\mathrm{HA}^{-2}$ & $\mathrm{~A}^{-3}$ & $\mathrm{H}_{2} \mathrm{~A}_{1}$ & $\mathrm{HA}_{1}^{-1}$ & $\mathrm{~A}_{1}^{-2}$ & $\mathrm{HA}_{2}$ & $\mathrm{~A}_{2}^{-1}$ & $\mathrm{OH}^{-1}$ \\
\hline $\mathrm{p} K_{1 \mathrm{~A}}$ & 1 & -1 & 1 & 0 & 0 & 0 & 0 & 0 & 0 & 0 \\
$\mathrm{p} K_{2 \mathrm{~A}}$ & 1 & 0 & -1 & 1 & 0 & 0 & 0 & 0 & 0 & 0 \\
$\mathrm{p} K_{3 \mathrm{~A}}$ & 1 & 0 & 0 & -1 & 1 & 0 & 0 & 0 & 0 & 0 \\
$\mathrm{p} K_{1 \mathrm{~A}_{1}}$ & 1 & 0 & 0 & 0 & 0 & -1 & 1 & 0 & 0 & 0 \\
$\mathrm{p} K_{2 \mathrm{~A}_{1}}$ & 1 & 0 & 0 & 0 & 0 & 0 & -1 & 1 & 0 & 0 \\
$\mathrm{p} K_{1 \mathrm{~A}_{2}}$ & 1 & 0 & 0 & 0 & 0 & 0 & 0 & 0 & -1 & 0 \\
$\mathrm{p} K_{\mathrm{w}}$ & 1 & 0 & 0 & 0 & 0 & 0 & 0 & 0 & 0 & 0 \\
\hline
\end{tabular}

TABLE 3: The experimental $\mathrm{pK}$ a data obtained for the mixture of PhA, Py4CA, and MA at various compositions of titrand D.

\begin{tabular}{|c|c|c|c|c|}
\hline Composition of D & $\mathrm{p} K_{n}$ & $\mathrm{p} K_{\mathrm{aPhA}} \pm s$ & $\mathrm{p} K_{\mathrm{aPy} 4 \mathrm{CA}} \pm s$ & $\mathrm{p} K_{\mathrm{aMA}} \pm s$ \\
\hline \multirow{2}{*}{$\begin{array}{l}\mathrm{PhA}+\mathrm{Py} 4 \mathrm{CA}+\mathrm{MA} \\
(1: 1: 1)\end{array}$} & $\mathrm{p} K_{1}$ & $2.97 \pm 0.07$ & - & $3.30 \pm 0.03$ \\
\hline & $\mathrm{p} K_{2}$ & $5.31 \pm 0.04$ & $4.69 \pm 0.03$ & - \\
\hline \multirow{2}{*}{$\begin{array}{l}\mathrm{PhA}+\mathrm{Py} 4 \mathrm{CA}+\mathrm{MA} \\
(1: 2: 2)\end{array}$} & $\mathrm{p} K_{1}$ & $2.81 \pm 0.06$ & - & $3.12 \pm 0.03$ \\
\hline & $\mathrm{p} K_{2}$ & $5.45 \pm 0.03$ & $4.71 \pm 0.01$ & - \\
\hline $\begin{array}{l}\mathrm{PhA}+\mathrm{Py} 4 \mathrm{CA}+\mathrm{MA} \\
(1: 2: 1)\end{array}$ & $\begin{array}{l}\mathrm{p} K_{1} \\
\mathrm{p} K_{2}\end{array}$ & $\begin{array}{l}2.78 \pm 0.04 \\
5.53 \pm 0.04\end{array}$ & $4.76 \pm 0.02$ & $3.4^{*}$ \\
\hline
\end{tabular}

${ }^{*} \mathrm{p} K_{\mathrm{a}}$ value const. taken from the literature [see Table 11].

TABLE 4: The experimental $\mathrm{p} K_{\mathrm{a}}$ data obtained for the mixture of $\mathrm{A}+\mathrm{MA}+\mathrm{PhA}+\mathrm{Py} 3 \mathrm{CA}$ and 2,6PyDCA + Py3CA.

\begin{tabular}{|c|c|c|c|c|c|}
\hline Composition of D & $\mathrm{p} K_{n}$ & $\mathrm{p} K_{\mathrm{aPhA}} \pm s$ & $\mathrm{p} K_{\mathrm{aPy} 3 \mathrm{CA}} \pm s$ & $\mathrm{p} K_{\mathrm{aMA}} \pm s$ & $\mathrm{p} K_{\mathrm{aA}} \pm s$ \\
\hline \multirow{2}{*}{$\mathrm{A}+\mathrm{MA}+\mathrm{PhA}+\mathrm{Py} 3 \mathrm{CA}$} & $\mathrm{p} K_{1}$ & $3.11 \pm 0.05$ & - & $3.74 \pm 0.03$ & $4.68 \pm 0.02$ \\
\hline & $\mathrm{p} K_{2}$ & $5.62 \pm 0.03$ & $4.82^{*}$ & - & - \\
\hline Composition of D & $\mathrm{p} K_{n}$ & $\mathrm{p} K_{\mathrm{a} 2,6 \mathrm{PyDCA}} \pm s$ & $\mathrm{p} K_{\mathrm{aPy} 3 \mathrm{CA}} \pm s$ & - & - \\
\hline \multirow{2}{*}{ 2,6PyDCA + Py3CA } & $\mathrm{p} K_{1}$ & $2.41 \pm 0.04$ & - & - & - \\
\hline & $\mathrm{p} K_{2}$ & $4.72 \pm 0.02$ & $5.19 \pm 0.04$ & - & - \\
\hline Composition of D & $\mathrm{p} K_{n}$ & $\mathrm{p} K_{\mathrm{aPhA}} \pm s$ & $\mathrm{p} K_{\mathrm{aPy} 3 \mathrm{CA}} \pm s$ & $\mathrm{p} K_{\mathrm{aCA}} \pm s$ & - \\
\hline \multirow{3}{*}{$\mathrm{PhA}+\mathrm{Py} 3 \mathrm{CA}+\mathrm{CA}$} & $\mathrm{p} K_{1}$ & $3.12 \pm 0.03$ & - & $3.04 \pm 0.03$ & - \\
\hline & $\mathrm{p} K_{2}$ & $5.53 \pm 0.03$ & $4.82^{*}$ & $4.46 \pm 0.05$ & - \\
\hline & $\mathrm{p} K_{3}$ & - & - & $6.05 \pm 0.04$ & - \\
\hline
\end{tabular}

${ }^{*} \mathrm{p} K_{\mathrm{a}}$ value const. from the literature [see Table 11].

the Lauda E100 circulation thermostat. The electrode was calibrated with the use of buffer solutions: potassium hydrogen phthalate ( $\mathrm{pH} 4.00)$, citric acid/ $\mathrm{Na}_{2} \mathrm{HPO}_{4}(\mathrm{pH} 7.00)$, and boric acid/KCl/NaOH (pH 10.00).

Titrant T $(0.1 \mathrm{~mol} / \mathrm{L} \mathrm{NaOH})$ was standardized according to the general analytical procedure and protected from carbon dioxide. Double distilled water of conductivity approximately $0.18 \mu \mathrm{S} / \mathrm{cm}$ was used throughout for the preparation of aqueous solutions of organic acids and bases under study. It was freshly produced in order to avoid carbon dioxide absorption. Other reagents together with their abbreviations used in the text are listed in Abbreviations section.

2.2. Analytical Procedure. Volume $V_{0}$ of $4.0 \mathrm{~mL}$ to $5.0 \mathrm{~mL}$ of titrand (D) was titrated with $0.1 \mathrm{~mol} \cdot \mathrm{L}^{-1}$ of titrant (T) using a Cerko Lab System, equipped with a syringe pump. Titrant (T) was added to titrand (D) in increments of $0.01 \mathrm{~mL}$, with a pause of $7 \mathrm{~s}$. The $\mathrm{p} K_{\mathrm{a}}$ values were calculated from the experimental data points $\left\{\left(V_{j}, \mathrm{pH}_{j}\right) \mid j=1, \ldots, N\right\}$ according to the Kostrowicki and Liwo algorithm $[9,10]$.

\section{Results and Discussion}

3.1. Carboxylic Acids Mixture. The representative $\mathrm{pH}$ titration curves for the mixture of carboxylic acids PhA : Py-4CA : MA are presented in Figures 1 and 2. Compositions of titrand D for the mixture (PhA : Py-4CA : MA) were as follows: $1: 1: 1$, $1: 2: 1,1: 2: 2$, and $1: 1: 1$ with 0.5 mole ratio of $\mathrm{HCl}$. The titration and fitted curves $\mathrm{pH}=f\left(V_{\mathrm{NaOH}}\right)$ obtained for mixture with molar ratio of the components $1: 2: 2$ (PhA : Py4CA : MA) are shown in Figure 2. The $\mathrm{p} K_{\mathrm{a}}$ data determined for the mixtures of carboxylic acids under study are listed in Tables 3 and 4 .

3.2. Organic Bases in Protonated Form (Cationic Acids). The amino group is one of the most fundamental functional groups considered in organic and pharmaceutical chemistry 
TABLE 5: The $\mathrm{p} K_{\mathrm{a}}$ 's values of compounds determined in the multicomponent mixture of amines, heterocyclic moiety, and weak (Mes) and strong $(\mathrm{HCl})$ acids at $25^{\circ} \mathrm{C}$.

\begin{tabular}{|c|c|c|c|c|}
\hline Composition of D & $\mathrm{p} K_{\mathrm{A}} \pm s$ & $\mathrm{pK}_{4 \mathrm{NH}_{2} \mathrm{Py}} \pm s$ & $\mathrm{p} K_{2 \mathrm{NH}_{2} \mathrm{Py}} \pm s$ & $\mathrm{p} K_{\mathrm{FA}} \pm s$ \\
\hline $\mathrm{A}+\mathrm{HCl}$ & $4.650 \pm 0.02$ & - & - & - \\
\hline $\mathrm{A}+4 \mathrm{NH}_{2} \mathrm{Py}+\mathrm{HCl}$ & $4.53 \pm 0.04$ & $9.27 \pm 0.18$ & - & - \\
\hline $\mathrm{A}+4 \mathrm{NH}_{2} \mathrm{Py}+2 \mathrm{NH}_{2} \mathrm{Py}+\mathrm{HCl}$ & $5.13 \pm 0.07$ & $9.59 \pm 0.07$ & $7.18 \pm 0.07$ & - \\
\hline $\mathrm{A}+2 \mathrm{NH}_{2} \mathrm{Py}+\mathrm{FA}$ & $4.7^{*}$ & - & $6.7^{*}$ & $\begin{array}{l}3.05 \pm 0.04 \\
4.26 \pm 0.02\end{array}$ \\
\hline Composition of D & $\mathrm{p} K_{\mathrm{Mes}} \pm s$ & $\mathrm{p} K_{2 \mathrm{NH}_{2} \mathrm{Py}} \pm s$ & $\begin{array}{c}\mathrm{p} K_{\mathrm{Py} 3 \mathrm{CA}} \pm s \\
\text { or } \mathrm{p} K_{\mathrm{Py} 4 \mathrm{CA}} \pm s\end{array}$ & $\begin{array}{c}\mathrm{p} K_{\mathrm{Bt}} \pm s \\
\text { or } \mathrm{p} K_{\mathrm{Bi}} \pm s\end{array}$ \\
\hline $\mathrm{Mes}+\mathrm{Bt}$ & $6.24 \pm 0.05$ & - & - & $8.75 \pm 0.59$ \\
\hline $\mathrm{Mes}+2 \mathrm{NH}_{2} \mathrm{Py}+\mathrm{Bi}+\mathrm{HCl}$ & $6.28 \pm 0.05$ & $7.22 \pm 0.05$ & - & $5.36 \pm 0.04$ \\
\hline $\mathrm{Mes}+2 \mathrm{NH}_{2} \mathrm{Py}+\mathrm{Py} 3 \mathrm{CA}$ & $6.29 \pm 0.03$ & $7.22 \pm 0.03$ & $4.78 \pm 0.03$ & - \\
\hline $\mathrm{Mes}+2 \mathrm{NH}_{2} \mathrm{Py}+\mathrm{Py} 4 \mathrm{CA}$ & $6.29 \pm 0.34$ & $6.39 \pm 0.34$ & $4.76 \pm 0.30$ & - \\
\hline Composition of D & $\mathrm{p} K_{\mathrm{ASA}} \pm s$ & $\mathrm{p} K_{2 \mathrm{NH}_{2} \mathrm{Py}} \pm s$ & $\mathrm{p} K_{\mathrm{Py} 3 \mathrm{CA}} \pm s$ & $\begin{array}{c}\mathrm{p} K_{\mathrm{AA}} \pm s \\
\text { or } \mathrm{p} K_{3 \mathrm{MePy}}\end{array}$ \\
\hline $\mathrm{ASA}+\mathrm{Py} 3 \mathrm{CA}+\mathrm{AA}$ & $3.66 \pm 0.05$ & - & $5.02 \pm 0.04$ & $4.15 \pm 0.05$ \\
\hline $\mathrm{ASA}+2 \mathrm{NH}_{2} \mathrm{Py}+3 \mathrm{MePy}$ & $3.61 \pm 0.03$ & $7.02 \pm 0.03$ & - & $5.82 \pm 0.03$ \\
\hline
\end{tabular}

${ }^{*} \mathrm{p} K_{\mathrm{a}}$ value const. from the literature [see Table 11].

TABLE 6: The $\mathrm{p} K_{\mathrm{a}}$ 's values of compounds determined in the mixture containing four-weak electrolytes (acids and bases) and a strong acid $(\mathrm{HCl})$ at $25^{\circ} \mathrm{C}$.

\begin{tabular}{llcccc}
\hline Composition of D & $\mathrm{p} K_{n}$ & $\mathrm{p} K_{\mathrm{PhA}} \pm s$ & $\mathrm{p} K_{\mathrm{MA}} \pm s$ & $\mathrm{p} K_{\mathrm{Py} 3 \mathrm{CA}} \pm s$ & $\mathrm{p} K_{\mathrm{A}} \pm \mathrm{s}$ \\
\hline $\mathrm{PhA}+\mathrm{MA}+\mathrm{Py} 3 \mathrm{CA}+\mathrm{A}+\mathrm{HCl}$ & $\mathrm{p} K_{1}$ & $3.11 \pm 0.05$ & $3.74 \pm 0.03$ & $-8^{*}$ & - \\
\hline Composition of D & $\mathrm{p} K_{2}$ & $5.62 \pm 0.03$ & - & $\mathrm{p}$ & - \\
\hline $\mathrm{AA}+\mathrm{Bi}+\mathrm{ImH}+\mathrm{BtH}+\mathrm{HCl}$ & $\mathrm{p} K_{n}$ & $\mathrm{p} K_{\mathrm{AA}} \pm s$ & $\mathrm{p} K_{\mathrm{Bi}} \pm s$ & $\mathrm{p} K_{\mathrm{ImH}} \pm s$ & $\mathrm{p} K_{\mathrm{BtH}} \pm s$ \\
\hline
\end{tabular}

${ }^{*} \mathrm{p} K_{\mathrm{a}}$ value const. from the literature [see Table 11].

TABLE 7: The $\mathrm{p} K_{\mathrm{a}}$ 's values of compounds determined in the multicomponent mixture of amino acid, heterocyclic moiety, and weak (Mes) and strong $(\mathrm{HCl})$ acids at $25^{\circ} \mathrm{C}$.

\begin{tabular}{|c|c|c|c|c|c|}
\hline Composition of D & $\mathrm{p} K_{n}$ & $\mathrm{p} K_{\mathrm{His}} \pm s$ & $\mathrm{p} K_{\mathrm{Py} 3 \mathrm{CA}}$ & $\mathrm{p} K_{\mathrm{Mes}}$ & $\mathrm{p} K_{\mathrm{ImH}}$ \\
\hline $\mathrm{L}-\mathrm{His}$ & $\mathrm{p} K_{3}$ & $9.67 \pm 0.01$ & - & - & - \\
\hline \multirow{2}{*}{ L-His $+\operatorname{HCl}(1: 1)$} & $\mathrm{p} K_{2}$ & $6.28 \pm 0.01$ & - & - & - \\
\hline & $\mathrm{p} K_{3}$ & $9.97 \pm 0.01$ & - & - & - \\
\hline \multirow{3}{*}{ L-His $+\mathrm{HCl}(1: 2)$} & $\mathrm{p} K_{1}$ & $1.54 \pm 0.04$ & - & - & - \\
\hline & $\mathrm{p} K_{2}$ & $6.26 \pm 0.01$ & - & - & - \\
\hline & $\mathrm{p} K_{2}$ & $9.66 \pm 0.01$ & - & - & - \\
\hline \multirow{3}{*}{$\begin{array}{l}\mathrm{L}-\mathrm{His}+\mathrm{Py} 3 \mathrm{CA}+ \\
+\mathrm{Mes}+\mathrm{ImH}\end{array}$} & $\mathrm{p} K_{1}$ & - & - & $6.28^{*}$ & $7.56^{*}$ \\
\hline & $\mathrm{p} K_{2}$ & $6.28^{*}$ & $4.68 \pm 0.24$ & - & - \\
\hline & $\mathrm{p} K_{3}$ & $10.06 \pm 0.55$ & - & - & - \\
\hline \multirow{3}{*}{$\begin{array}{l}\mathrm{L}-\mathrm{His}+\mathrm{Py} 3 \mathrm{CA}+ \\
+\mathrm{Mes}+\mathrm{ImH}+\mathrm{HCl}\end{array}$} & $\mathrm{p} K_{1}$ & - & $4.81 \pm 0.28$ & $6.07 \pm 0.14$ & $7.49 \pm 0.05$ \\
\hline & $\mathrm{p} K_{2}$ & $6.43 \pm 0.74$ & - & - & - \\
\hline & $\mathrm{p} K_{3}$ & $10.06 \pm 0.37$ & - & - & - \\
\hline Composition of D & $\mathrm{p} K_{n}$ & $\mathrm{p} K_{\mathrm{Ala}}$ & - & - & - \\
\hline L-Ala & $\mathrm{p} K_{2}$ & $10.30 \pm 0.01$ & - & - & - \\
\hline \multirow{2}{*}{$\mathrm{L}-\mathrm{Ala}+\mathrm{HCl}(1: 1)$} & $\mathrm{p} K_{1}$ & $2.25 \pm 0.01$ & - & - & - \\
\hline & $\mathrm{p} K_{2}$ & $10.25(0.07)$ & - & - & - \\
\hline
\end{tabular}

${ }^{*} \mathrm{p} K_{\mathrm{a}}$ value const. from the literature [see Table 11]. 
TABLE 8: The $\mathrm{p} K_{\mathrm{a}}$ 's values of compounds determined in the multicomponent mixture of phenol and enol OH-acids together with other weak electrolytes at $25^{\circ} \mathrm{C}$.

\begin{tabular}{|c|c|c|c|c|}
\hline Composition of D & $\mathrm{p} K_{\mathrm{A}} \pm s$ & $\mathrm{p} K_{\mathrm{FA}} \pm s$ & $\mathrm{p} K_{4 \mathrm{NO}_{2} \mathrm{PhOH}} \pm s$ & - \\
\hline $\mathrm{A}+\mathrm{FA}+4 \mathrm{NO}_{2} \mathrm{PhOH}$ & $4.87 \pm 0.02$ & $\begin{array}{l}3.05 \pm 0.03 \\
4.06 \pm 0.03\end{array}$ & $7.42 \pm 0.04$ & - \\
\hline Composition of D & $\mathrm{p} K_{\mathrm{MAL}} \pm s$ & $\mathrm{p} K_{\mathrm{FA}} \pm s$ & $\mathrm{p} K_{4 \mathrm{NO}_{2} \mathrm{PhOH}} \pm s$ & - \\
\hline $\mathrm{FA}+\mathrm{MAL}+4 \mathrm{NO}_{2} \mathrm{PhOH}$ & $\begin{array}{l}3.59 \pm 0.04 \\
5.14 \pm 0.02\end{array}$ & $\begin{array}{l}2.50 \pm 0.04 \\
4.06 \pm 0.04\end{array}$ & $7.4 \pm 0.03$ & - \\
\hline Composition of D & $\mathrm{p} K_{\mathrm{AA}} \pm s$ & $\mathrm{p} K_{\mathrm{Mes}} \pm s$ & $\mathrm{p} K_{\mathrm{Pcm}} \pm s$ & - \\
\hline $\mathrm{AA}+\mathrm{Mes}+\mathrm{Pcm}$ & $4.14 \pm 0.03$ & $6.24 \pm 0.04$ & $9.95 \pm 0.05$ & - \\
\hline Composition of D & $\mathrm{p} K_{\mathrm{ASA}} \pm s$ & $\mathrm{p} K_{\mathrm{FA}} \pm s$ & $\mathrm{p} K_{4 \mathrm{NO}_{2} \mathrm{PhOH}} \pm s$ & $\mathrm{p} K_{\mathrm{Kpf}}$ \\
\hline $\mathrm{ASA}+\mathrm{FA}+4 \mathrm{NO}_{2} \mathrm{PhOH}+\mathrm{Kpf}$ & $3.77 \pm 0.05$ & $\begin{array}{l}2.91 \pm 0.06 \\
4.75 \pm 0.37\end{array}$ & $7.52 \pm 0.05$ & $4.68 \pm 0.36$ \\
\hline
\end{tabular}

TABLE 9: The $\mathrm{p} K_{\mathrm{a}}$ 's values of compounds determined in the multicomponent mixture which comprises barbituric acid, $2(1 \mathrm{H})$ pyrazylidene acetonitrile, and phthalic acid at $25^{\circ} \mathrm{C}$.

\begin{tabular}{lcccc}
\hline $\begin{array}{l}\text { Composition } \\
\text { of D }\end{array}$ & $\mathrm{p} K_{n}$ & $\mathrm{p} K_{2(1 \mathrm{H}) \mathrm{PyAN}} \pm s$ & $\mathrm{p} K_{\mathrm{BA}} \pm s$ & $\mathrm{p} K_{\mathrm{PhA}} \pm s$ \\
\hline $2(1 \mathrm{H}) \mathrm{PyAN}+$ & $\mathrm{p} K_{1}$ & $7.10 \pm 0.09$ & $4.01 \pm 0.04$ & $2.39 \pm 0.08$ \\
$\mathrm{BA}+\mathrm{PhA}$ & $\mathrm{p} K_{2}$ & - & - & $5.76 \pm 0.05$ \\
\hline
\end{tabular}

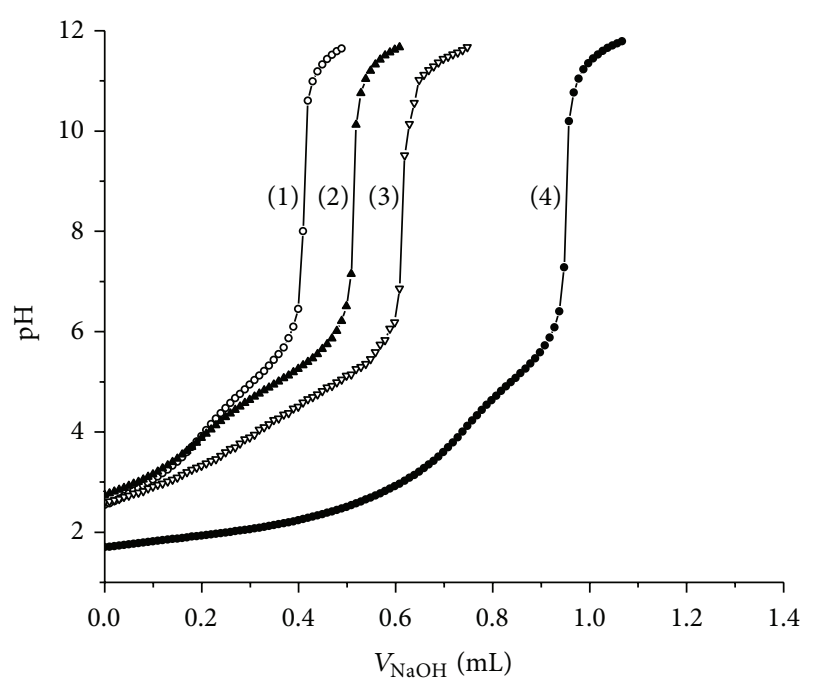

(1) PhA : Py-4CA : MA $1: 1: 1$

$\rightarrow-(2) \mathrm{PhA}: \mathrm{Py}-4 \mathrm{CA}: \mathrm{MA} 1: 2: 1$

$\rightarrow-(3) \mathrm{PhA}: \mathrm{Py}-4 \mathrm{CA}: \mathrm{MA} 1: 2: 2$

$\rightarrow-(4) \mathrm{PhA}: \mathrm{Py}-4 \mathrm{CA}: \mathrm{MA} 1: 1: 1$ and $0.5 \mathrm{HCl}$

FIGURE 1: Titration curves $\mathrm{pH}=f\left(V_{\mathrm{NaOH}}\right)$ obtained for the mixture of $\mathrm{PhA}, \mathrm{Py} 4 \mathrm{CA}$, and MA with different mole ratio of acids and with excess of $\mathrm{HCl}$.

and its $\mathrm{p} K_{\mathrm{a}}$ is an important and extensively studied property. The $\mathrm{p} K_{\mathrm{a}}$ value of the amino group can vary over several orders of magnitude (ammonia, $\mathrm{p} K_{\mathrm{a}}=9.26$; aniline, $\mathrm{p} K_{\mathrm{a}}=4.63$ [11]), depending on its chemical environment. In our study the $\mathrm{p} K_{\mathrm{a}}$ refers to the conjugate acid $\mathrm{B}+\mathrm{H}^{+1}=\mathrm{BH}^{+1}$ and dissociation according to the scheme: $\mathrm{BH}^{+1}=\mathrm{B}+\mathrm{H}^{+1}$.

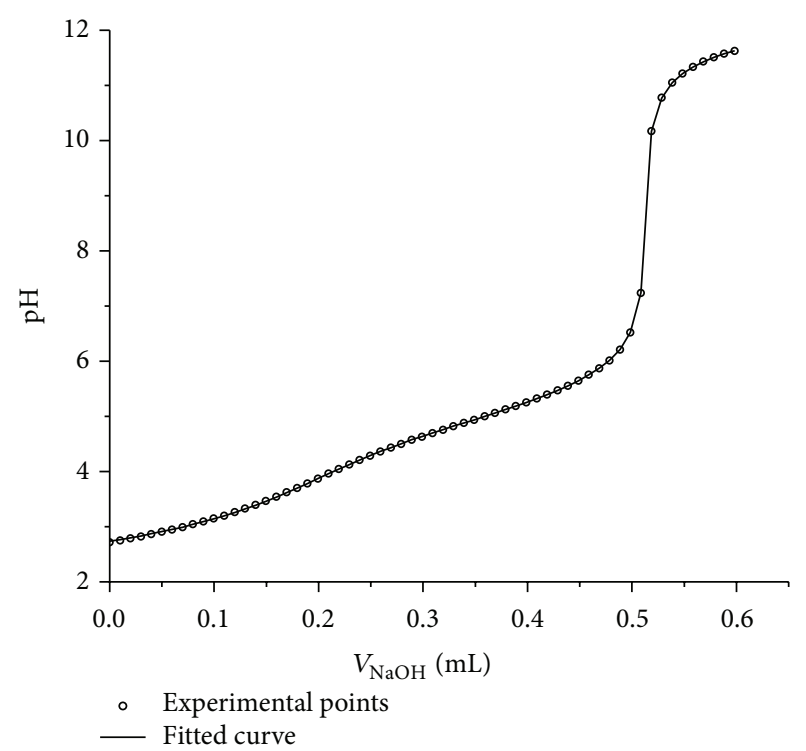

FIgURE 2: Titration and fitted curves $\mathrm{pH}=f\left(V_{\mathrm{NaOH}}\right)$ obtained for mixture of $\mathrm{PhA}, \mathrm{Py}-4 \mathrm{CA}$, and MA with mole ratio of acids $1: 2: 2$.

We have tested a mixture of organic bases exemplified by aniline and pyridine derivatives ( 2 - and 4 -substituted aminopyridines and methylpyridine) at the presence of equimolar ratio of $\mathrm{HCl}$. Organic bases exist in the system in the protonated form (cationic acids, $\mathrm{BH}^{+1}$ ) and dissociate according to the scheme: $\mathrm{BH}^{+1}=\mathrm{B}+\mathrm{H}^{+1},{K_{\mathrm{BH}}}^{+}=[\mathrm{B}]\left[\mathrm{H}^{+1}\right] /$ $\left[\mathrm{BH}^{+1}\right]$.

Based on the titration curve of mixture of amines (presented in the form of cationic acids), the $\mathrm{p} K_{\mathrm{a}}$ values were determined for aniline (B) and for 2- and 4-aminopyridine (2- $\left.\mathrm{NH}_{2} \mathrm{Py}, 4-\mathrm{NH}_{2} \mathrm{Py}\right)$. The heterocyclic five-membered ring systems of imidazole (Im), benzotriazole (Bt), and benzimidazole $(\mathrm{Bi})$ were also investigated. We have applied a general procedure for the titration of mixtures of different types (and concentration) of organic acids and bases. The elaborated procedure was also tested in the presence of biological buffers exemplified by 2-(N-morpholino) ethane-sulfonic acid (Mes) [12]. 
TABLE 10: The $\mathrm{p} K_{\mathrm{a}}$ 's values of compounds with pharmaceutical importance determined in the mixture containing other weak electrolytes $25^{\circ} \mathrm{C}$.

\begin{tabular}{|c|c|c|c|c|c|}
\hline Composition of D & $\mathrm{p} K_{\mathrm{AA}} \pm s$ & $\mathrm{p} K_{\text {Mes }} \pm s$ & $\mathrm{p} K_{\mathrm{Pcm}} \pm s$ & $\mathrm{p} K_{\mathrm{ASA}}$ & $\mathrm{p} K_{\mathrm{CA}}$ \\
\hline $\mathrm{AA}+\mathrm{Mes}+\mathrm{Pcm}$ & $4.14 \pm 0.03$ & $6.24 \pm 0.04$ & $9.95 \pm 0.05$ & - & - \\
\hline Composition of D & $\mathrm{p} K_{\mathrm{ASA}} \pm s$ & $\mathrm{p} K_{\mathrm{ImH}} \pm s$ & $\mathrm{p} K_{\mathrm{Eph}} \pm s$ & - & - \\
\hline $\mathrm{ASA}+\mathrm{ImH}+\mathrm{Eph} * \mathrm{HCl}$ & $3.49 \pm 0.03$ & $7.21 \pm 0.06$ & $9.94 \pm 0.05$ & - & - \\
\hline Composition of D & $\mathrm{p} K_{\mathrm{Met}} \pm s$ & $\mathrm{p} K_{\mathrm{ImH}} \pm s$ & $\mathrm{p} K_{\mathrm{Mes}} \pm s$ & - & - \\
\hline Met + ImH + Mes & $2.28 \pm 0.04$ & $7.35 \pm 0.02$ & $6.19 \pm 0.01$ & - & - \\
\hline Composition of D & $\mathrm{p} K_{\mathrm{ASA}} \pm s$ & $\mathrm{p} K_{\mathrm{Mes}} \pm s$ & $\mathrm{p} K_{\mathrm{L}-\mathrm{His}} \pm \mathrm{s}$ & - & - \\
\hline $\mathrm{ASA}+\mathrm{Mes}+\mathrm{L}-\mathrm{His}$ & $3.57 \pm 0.03$ & $6.29 \pm 0.03$ & $\begin{array}{c}\mathrm{p} K_{1 \mathrm{~L}-\mathrm{His}}=1.54^{*} \\
\mathrm{p} K_{2 \mathrm{~L}-\mathrm{His}}=5.96 \pm 0.05 \\
\mathrm{p} K_{3 \mathrm{~L}-\mathrm{His}}=9.67 \pm 0.59\end{array}$ & - & - \\
\hline Composition of D & $\mathrm{p} K_{\mathrm{KTL}} \pm s$ & $\mathrm{p} K_{\mathrm{Mes}} \pm s$ & $\mathrm{p} K_{\mathrm{L}-\mathrm{His}} \pm s$ & - & - \\
\hline $\mathrm{KTL}+\mathrm{Mes}+\mathrm{L}-\mathrm{His}$ & $\begin{array}{c}\mathrm{p} K_{1} 3.09 \pm 0.05 \\
\mathrm{p} K_{2} 6.15^{*}\end{array}$ & $6.15^{*}$ & $\begin{array}{c}\mathrm{p} K_{2 \mathrm{~L}-\mathrm{His}}=6.15^{*} \\
\mathrm{p} K_{3 \mathrm{~L}-\mathrm{His}}=9.58 \pm 0.06\end{array}$ & - & - \\
\hline Composition of D & $\mathrm{p} K_{\mathrm{AA}} \pm s$ & $\mathrm{p} K_{\mathrm{Mes}} \pm s$ & $\mathrm{p} K_{\mathrm{Ppv}} \pm s$ & $\mathrm{p} K_{\mathrm{ASA}}$ & $\mathrm{p} K_{\mathrm{CA}}$ \\
\hline $\mathrm{AA}+\mathrm{Mes}+\mathrm{Ppv}$ & $4.24 \pm 0.02$ & $6.55 \pm 0.03$ & $6.02 \pm 0.06$ & - & - \\
\hline $\mathrm{AA}+\mathrm{Mes}+\mathrm{ASA}+\mathrm{CA}$ & $4.05 \pm 0.16$ & - & - & $3.90 \pm 0.16$ & $\begin{array}{c}\mathrm{p} K_{1}=2.53 \pm 0.30 \\
\mathrm{p} K_{2}=5.03 \pm 0.33 \\
\mathrm{p} K_{3}=6.5 \pm 0.34\end{array}$ \\
\hline
\end{tabular}

${ }^{*} \mathrm{p} K_{\mathrm{a}}$ value const. from the literature [see Table 11].

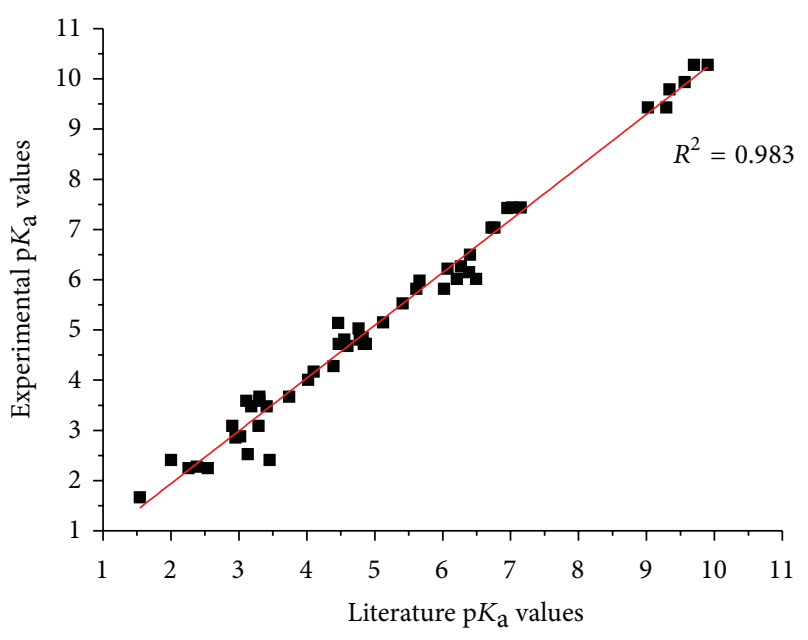

FIGURE 3: Plot of experimental versus literature $\mathrm{p} K_{\mathrm{a}}$ 's values for the compounds under study.

The values of dissociation constants obtained for mixtures of different type of bases and acids are listed in Tables 5 and 6.

3.3. Mixtures of Amino acids with Organic Acids and Bases. The presented general procedure was applied for studying the system consisting of amino acids, organic acids, and bases. The $\mathrm{p} K_{\mathrm{a}}$ values of this type of mixtures were calculated based on a single titration curve. Experimental results confirm the general application of the proposed procedure for the determination of $\mathrm{p} K_{\mathrm{a}}$ value for mixtures of any degree of complexity composed of weak acids and bases. The $\mathrm{p} K_{\mathrm{a}}$ values of weak acids $\left(\mathrm{H}_{n} \mathrm{~A}\right)$, bases $(\mathrm{B})$, and amino acids $\left(\mathrm{AB}^{ \pm}\right)$ in the mixture of these types of components were determined. Composition of the tested mixtures (titrand D) and $\mathrm{p} K_{\mathrm{a}}$ values are listed in Table 7.

3.4. Phenol and Enol OH-Acids as Components of Titrand D. The acidity of the phenol group (OH-acid) depends on the substituent of the aromatic ring and its $\mathrm{p} K_{\mathrm{a}}$ ranges from 4 to 11 [8]. We have performed the titration and relevant calculations for several mixtures of phenolic compounds, exemplified by $4-\mathrm{NO}_{2}$ phenol and a drug $\mathrm{N}$-(4-hydroxyphenyl)acetamide (paracetamol) with different type of organic acids and bases as titrands D. The results are summarised in Table 8 .

3.5. Heterocyclic $N-H$-Acids as Components of Titrand D. The barbituric acid (BA) and a new class of $2(1 \mathrm{H})$-pyrazylidene acetonitrile derivatives $(2(1 \mathrm{H})$ PyAN), with marked pharmaceutical importance $[13,14]$, were tested at the presence of phthalic acid. Barbituric acid was also tested at the presence of different drugs (Table 9).

3.6. Determination of $\mathrm{p} K_{\mathrm{a}}$ Values for Different Drugs as a Components of Titrand D. For all tested compounds with pharmaceutical importance we confirmed that the elaborated method could be recommended as a general approach to the determination of $\mathrm{p} K_{\mathrm{a}}$ values for weak acids and bases in mixtures of any degree of complexity. The composition of titrand $\mathrm{D}$ and the $\mathrm{p} K_{\mathrm{a}}$ values determined for drugs under study are listed in Table 10. 
TABLE 11: Experimental and literature $\mathrm{p} K_{\mathrm{a}}$ 's values of the compounds under study.

\begin{tabular}{|c|c|c|c|c|}
\hline No. & Compounds & $\mathrm{p} K_{\exp }{ }^{*}$ & $\mathrm{p} K_{\text {literature }}$ & Reference \\
\hline 1 & A & 4.81 & $4.55-4.78$ & [11] \\
\hline 2 & AA & 4.17 & 4.10 & [15] \\
\hline 3 & ASA & 3.67 & $3.30-3.74$ & [16] \\
\hline 4 & BA & 4.01 & 4.02 & [17] \\
\hline 5 & $\mathrm{Bi}$ & 5.98 & 5.66 & [18] \\
\hline 6 & $\mathrm{CA}$ & $\begin{array}{l}2.53 \\
5.03 \\
6.50\end{array}$ & $\begin{array}{l}3.13 \\
4.76 \\
6.40\end{array}$ & [19] \\
\hline 7 & Eph & 9.94 & 9.56 & [20] \\
\hline 8 & FA & $\begin{array}{l}2.88 \\
4.28\end{array}$ & $\begin{array}{l}3.02 \\
4.39\end{array}$ & [21] \\
\hline 9 & $\mathrm{Im}$ & 7.43 & 6.95 & [22] \\
\hline 10 & KTL & $\begin{array}{l}3.09 \\
6.15\end{array}$ & $\begin{array}{c}2.90-3.29 \\
6.39\end{array}$ & [16] \\
\hline 11 & Ktp & 4.68 & 4.6 & [23] \\
\hline 12 & L-ala & $\begin{array}{c}2.25 \\
10.28\end{array}$ & $\begin{array}{c}2.26-2.54 \\
9.7-9.9\end{array}$ & [18] \\
\hline 13 & L-his & $\begin{array}{l}1.67 \\
6.22 \\
9.79\end{array}$ & $\begin{array}{l}1.54 \\
6.07 \\
9.34\end{array}$ & [22] \\
\hline 14 & MA & 3.48 & $3.18-3.41$ & {$[18]$} \\
\hline 15 & 3-MePy & 5.82 & $5.61-6.02$ & [18] \\
\hline 16 & MAL & $\begin{array}{l}3.59 \\
5.14\end{array}$ & $\begin{array}{l}3.11-3.30 \\
4.46-5.12\end{array}$ & [18] \\
\hline 17 & Mes & 6.27 & 6.27 & [22] \\
\hline 18 & Met & 2.28 & 2.38 & {$[16]$} \\
\hline 19 & $2-\mathrm{NH}_{2} \mathrm{Py}$ & 7.04 & $6.72-6.76$ & {$[16]$} \\
\hline 20 & $4-\mathrm{NH}_{2} \mathrm{Py}$ & 9.43 & $9.02-9.29$ & [16] \\
\hline 21 & $4-\mathrm{NO}_{2} \mathrm{PhOH}$ & 7.44 & $7.02-7.15$ & {$[11]$} \\
\hline 22 & $\mathrm{PhA}$ & $\begin{array}{l}2.86 \\
5.53\end{array}$ & $\begin{array}{l}2.95 \\
5.41\end{array}$ & [22] \\
\hline 23 & Ppv & 6.02 & $6.21-6.49$ & {$[16]$} \\
\hline 24 & 2(1H)PyAN & 7.10 & - & - \\
\hline 25 & Рy-3CA & 4.85 & 4.82 & [24] \\
\hline 26 & Py-4CA & 4.72 & 4.84 & [24] \\
\hline 27 & 2.6-PyDCA & $\begin{array}{l}2.41 \\
4.72\end{array}$ & $\begin{array}{l}2.00-3.45 \\
4.47-4.87\end{array}$ & [18] \\
\hline
\end{tabular}

\section{Conclusions}

A new approach for studying equilibrium constants for the dissociation of different types of weak electrolytes present in a mixture of any degree of complexity has been proposed. Potentiometric titration technique and numerical procedure based on an original algorithm elaborated by Kostrowicki and Liwo and adopted in the Cerko Lab software have successfully been applied to obtain the $\mathrm{p} K_{\mathrm{a}}$ values of a variety of classes of compounds comprising of common organic acids and bases, amino acids, phenols and enols $\mathrm{OH}$ acids, and heterocyclic $\mathrm{N}-\mathrm{H}$-acids as well as compounds of pharmaceutical importance. It was shown that the $\mathrm{p} K_{\mathrm{a}}$ values of the compound present in the mixture can be determined directly without the need to separate individual analytes. The obtained $\mathrm{p} K_{\mathrm{a}}$ values of the electrolytes under study are in a good agreement with those reported in the literature (Table 11, Figure 3). Thus, the presented methodology can be considered as a fast, simple, inexpensive, and reagents-saving way for studying equilibria in the mixture of electrolytes. Moreover, it does not require a highly trained personnel. The methodology described in this paper can be routinely used in a regular analytical practice.

\section{Abbreviations}

$C_{0}: \quad$ Concentration $[\mathrm{mol} / \mathrm{L}]$ of weak electrolyte

D: $\quad$ Titrand (solution titrated)

T: $\quad$ Titrant (titrating solution)

K: $\quad$ Dissociation constant for a weak electrolyte

$\mathrm{p} K=-\log K:$ Acidity parameter

$V: \quad$ Volume $[\mathrm{mL}]$ of $\mathrm{T}$

$V_{0}: \quad$ Volume $[\mathrm{mL}]$ of $\mathrm{D}$

A: $\quad$ Aniline, aminobenzene (Sigma

Aldrich $>99.5 \%$ )

AA: $\quad$ Ascorbic acid L(+), (5R)-[(1S)-1,2-dihydroxyethyl]-3,4dihydroxyfuran-2(5H)-one

(Chempur, p.a.)

ASA: $\quad$ Acetylsalicylic acid, 2-acetoxybenzoic acid (Sigma Aldrich, >99.5\%)

BA: $\quad$ Barbituric acid, pyrimidine-2,4,6(1H,3H,5H)-trione (Fluka, p.a.)

$\mathrm{Bi}: \quad 1 H$-Benzimidazole (Sigma Aldrich)

Bt: $\quad$ 1,2,3-Benzotriazole (Sigma Aldrich)

CA: $\quad$ Citric acid monohydrate, 2-hydroxypropane-1,2,3-tricarboxylic acid (P.P.H. Standard S. z o.o., p.a.)

Eph: $\quad$ Ephedrine hydrochloride, (R,S)-2(methylamino)-1-phenylpropan-1-ol

FA: $\quad$ Fumaric acid, $(E)$-butenedioic acid (Sigma Aldrich, >99\%)

Im: $\quad 1 H$-Imidazole (Sigma Aldrich)

Kpf: Ketoprofen,

(RS)-2-(3-benzoylphenyl)propanoic acid

KTL: $\quad$ Ketoconazole, $1-[4-(4-\{[(2 R, 4 S)-2-$

(2,4-dichlorophenyl)-2-(1H -imidazol-1-ylmethyl)-1,3-dioxolan4-yl]methoxy\} phenyl)piperazin-1yl]ethan-1-one

L-ala: $\quad$ L-Alanine, 2-aminopropanoic acid (Fluka)

L-his: $\quad$ L-Histidine, 2-amino-3-(1Himidazol-4-yl)propanoic acid (Sigma Aldrich)

MA: $\quad$ Mandelic acid, 2-hydroxy-2-phenylacetic acid (Alfa Aesar Gmbh \& Co, >99\%) 
MAL: $\quad$ Malic acid (hydroxybutanedioic acid)

Met: $\quad$ Metronidazole (2-(2-methyl-5-nitro-1H -imidazol-1-yl)ethanol)

3-MePy: 3-Methylpyridine (Sigma Aldrich)

Mes: 2-(N-Morpholino)ethanesulfonic acid hydrate (Sigma Aldrich, $\geq 99 \%$ )

2- $\mathrm{NH}_{2} \mathrm{Py}: \quad 2$-Aminopyridine (Sigma Aldrich)

4- $\mathrm{NH}_{2} \mathrm{Py}$ : 4-Aminopyridine (Sigma Aldrich)

4-NO $\mathrm{N}_{2} \mathrm{PhOH}$ : 4-Nitrophenol (POCH S.A.)

Ppv: Papaverine (1-(3,4-dimethoxybenzyl)-6,7dimethoxyisoquinoline)

Pcm: Paracetamol (N-(4-hydroxyphenyl)acetamide)

PhA: Phthalic acid, benzene-1,2-dicarboxylic acid (POCH S.A.)

2(1H)PyAN: $\alpha-2(1-\mathrm{H})$ pyrazylidene $\alpha$-2-cyanoethylacetate

Py-3CA: $\quad$ Pyridine-3-carboxylic acid (Sigma Aldrich, p.a.)

Py-4CA: $\quad$ Pyridine- 4-carboxylic acid (Sigma Aldrich, p.a.)

2,6-PyDCA: Pyridine-2,6-dicarboxylic acid (Sigma Aldrich, p.a.).

\section{Conflict of Interests}

The authors declare that there is no conflict of interests.

\section{References}

[1] J. I. Wells, Pharmaceutical Preformulation, Eills Haarwood Ltd., London, UK, 1998.

[2] D. T. Manallack, "The $\mathrm{p} K_{\mathrm{a}}$ distribution of drugs: application to drug discovery," Perspectives in Medicinal Chemistry, vol. 1, pp. 25-38, 2007.

[3] M. D. Liptak, K. C. Gross, P. G. Seybold, S. Feldgus, and G. C. Shields, "Absolute pKa determinations for substituted phenols," Journal of the American Chemical Society, vol. 124, no. 22, pp. 6421-6427, 2002.

[4] A. M. Toth, D. L. Liptak, D. L. Phillips, and G. C. Shields, "Accurate relative $\mathrm{pK}_{a}$ calculations for carboxylic acids using complete basis set and Gaussian- $n$ models combined with continuum solvation methods," The Journal of Chemical Physics, vol. 114, pp. 4595-4606, 2001.

[5] S. Jelfs, P. Ertl, and P. Selzer, "Estimation of pKa for druglike compounds using semiempirical and information-based descriptors," Journal of Chemical Information and Modeling, vol. 47, no. 2, pp. 450-459, 2007.

[6] J. Zhang, T. Kleinöder, and J. Gasteiger, "Prediction of $\mathrm{pKa}$ values for aliphatic carboxylic acids and alcohols with empirical atomic charge descriptors," Journal of Chemical Information and Modeling, vol. 46, no. 6, pp. 2256-2266, 2006.

[7] R. S. Vařeková, S. Geidl, C.-M. Ionescu et al., "Predicting $\mathrm{p} K_{a}$ values of substituted phenols from atomic charges: comparison of different quantum mechanical methods and charge distribution schemes," Journal of Chemical Information and Modeling, vol. 51, no. 8, pp. 1795-1806, 2011.

[8] A. A. Ibrahim and E. A. Abdalrazaq, "Physical properties of phenol compound: semi-empirical calculation of substituent effects," American Journal of Applied Sciences, vol. 6, no. 7, pp. 1385-1389, 2009.

[9] J. Kostrowicki and A. Liwo, "A general method for the determination of the stoichiometry of unknown species in multicomponent systems from physicochemical measurements," Computers and Chemistry, vol. 11, no. 3, pp. 195-210, 1987.

[10] J. Kostrowicki and A. Liwo, "Determination of equilibrium parameters by minimization of an extended sum of squares," Talanta, vol. 37, no. 6, pp. 645-650, 1990.

[11] L. G. Sillen, A. E. Martell, and J. Bjerrum, Stability Constants of Metal-Ion Complexes, supplement no. 1, part I, The Chemical Society, London, UK, 1971.

[12] N. E. Good, G. D. Winget, W. Winter, T. N. Connolly, S. Izawa, and R. M. M. Singh, "Hydrogen ion buffers for biological research," Biochemistry, vol. 5, no. 2, pp. 467-477, 1966.

[13] R. Kaliszan, B. Pilarski, K. Ośmiałowski, H. Strzałkowska-Grad, and E. Hać, "Analgesic activity of new pyrazine $\mathrm{CH}$ and $\mathrm{NH}$ acids and their Hydrophobic and electron donating properties," Pharmaceutisch Weekblad, vol. 7, pp. 141-145, 1985.

[14] J. Petrusewicz, M. Turowski, H. Foks, B. Pilarski, and R. Kaliszan, "Comparative studies of antiplatelet activity of nonsteroidal antiinflammatory drugs and new pyrazine $\mathrm{CH}$ - and NH-acids," Life Sciences, vol. 56, no. 9, pp. 667-677, 1995.

[15] D. D. Perrin, Dissociation Constants of Organic Bases in Aqueous Solution, Butterworths, Boston, Mass, USA, 1965, supplement, 1972.

[16] M. Shalaeva, J. Kenseth, F. Lombardo, and A. Bastin, "Measurement of dissociation constants ( $\mathrm{p} K_{a}$ values) of organic compounds by multiplexed capillary electrophoresis using aqueous and cosolvent buffers," Journal of Pharmaceutical Sciences, vol. 97, no. 7, pp. 2581-2606, 2008.

[17] A. Abbaspour and M. A. Kamyabi, "Acidity constants and thermodynamic parameters of barbituric and diethylbarbituric acids in water, (water + tetrahydrofuran ), and (water + triton X-100) systems," Journal of Chemical \& Engineering Data, vol. 46, no. 3, pp. 623-625, 2001.

[18] The Chemical Society, Stability Constants of Metal-Ion Complexes, Part B, The Chemical Society, London, UK, 1979.

[19] D. Wyrzykowski, J. Czupryniak, T. Ossowski, and L. Chmurzyński, "Thermodynamic interactions of the alkaline earth metal ions with citric acid," Journal of Thermal Analysis and Calorimetry, vol. 102, no. 1, pp. 149-154, 2010.

[20] G. Völgyi, R. Ruiz, K. Box, J. Comer, E. Bosch, and K. Takács-Novák, "Potentiometric and spectrophotometric $\mathrm{p} K_{a}$ determination of water-insoluble compounds: validation study in a new cosolvent system," Analytica Chimica Acta, vol. 583, no. 2, pp. 418-428, 2007.

[21] L. G. Sillén and A. E. Martell, Stability Constants of Metal-Ion Complexes, vol. 17 of Special Publication, The Chemical Society, London, UK, 1979.

[22] R. N. Goldberg, N. Kishore, and R. M. Lennen, "Thermodynamic quantities for the ionization reactions of buffers," Journal of Physical and Chemical Reference Data, vol. 31, no. 2, pp. 231$370,2002$.

[23] J. J. Sheng, N. A. Kasim, R. Chandrasekharan, and G. L. Amidon, "Solubilization and dissolution of insoluble weak acid, ketoprofen: effects of $\mathrm{pH}$ combined with surfactant," European Journal of Pharmaceutical Sciences, vol. 29, no. 3-4, pp. 306-314, 2006.

[24] R. Casasnovas, J. Frau, J. Ortega-Castro, A. Salvà, J. Donoso, and F. Muñoz, "Absolute and relative $\mathrm{p} K_{a}$ calculations of mono and diprotic pyridines by quantum methods," Journal of Molecular Structure: THEOCHEM, vol. 912, no. 1-3, pp. 5-12, 2009. 

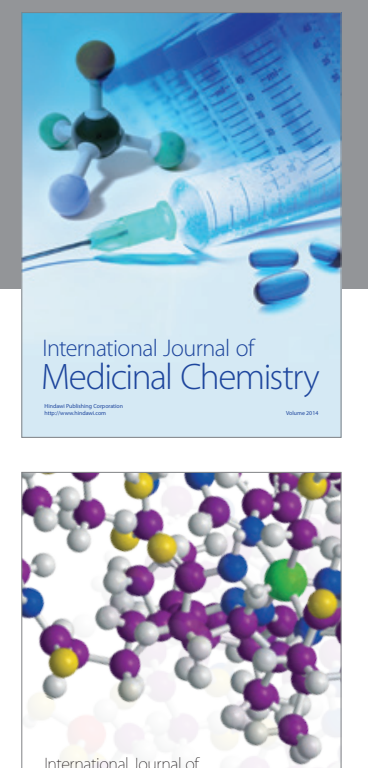

\section{Carbohydrate} Chemistry

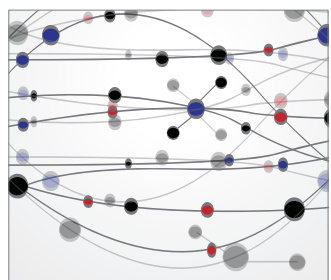

The Scientific World Journal
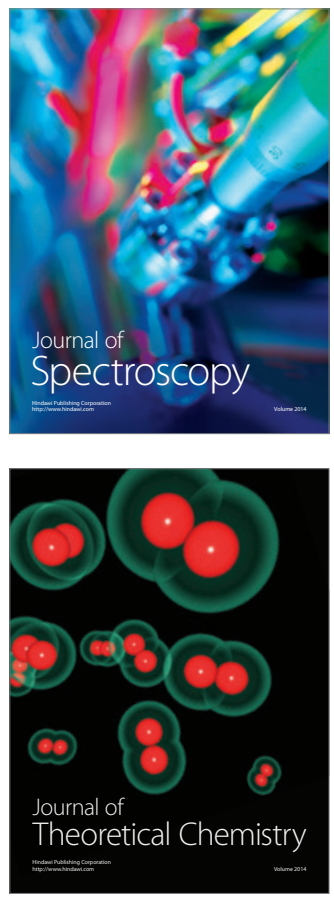
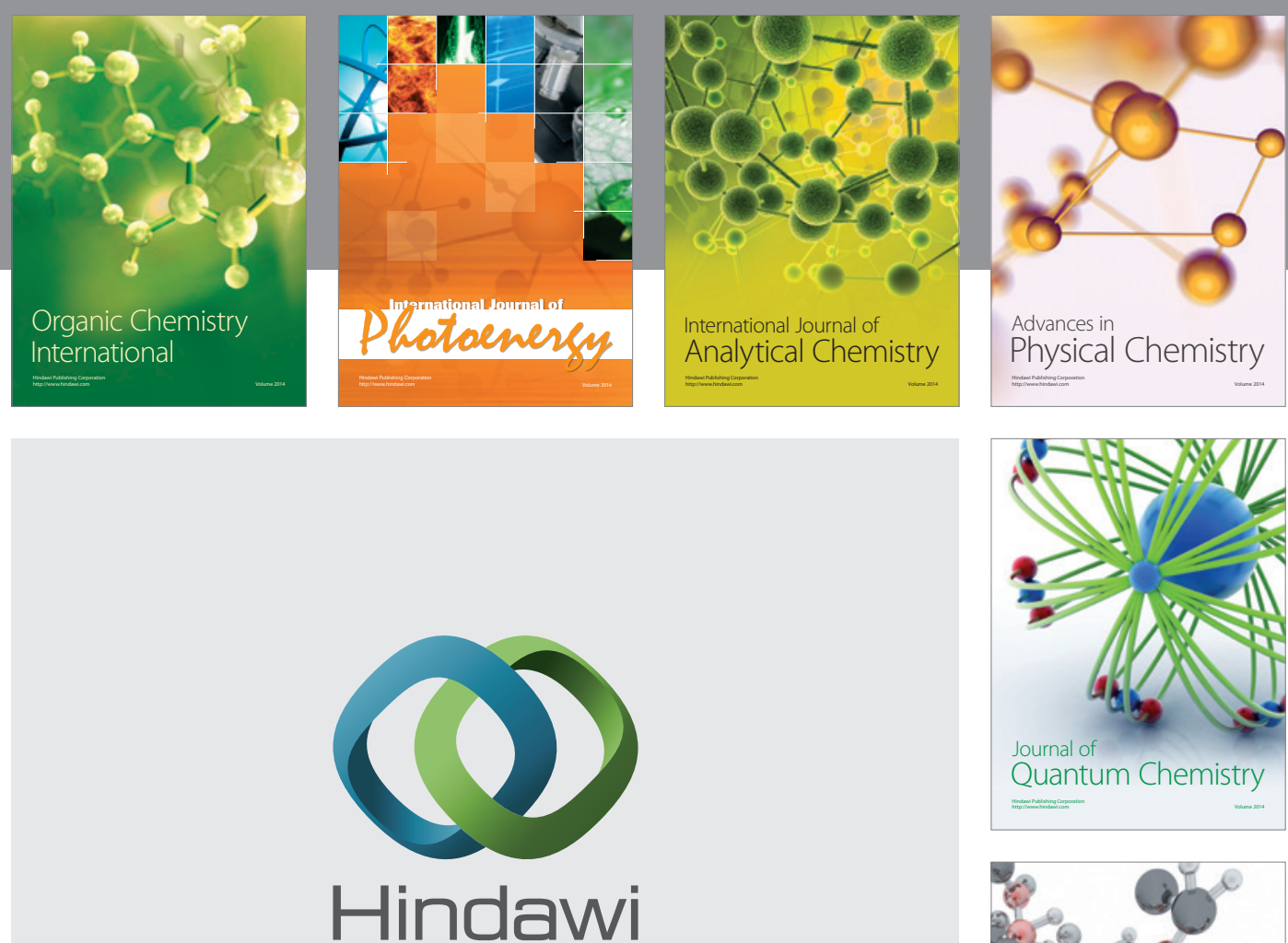

Submit your manuscripts at

http://www.hindawi.com

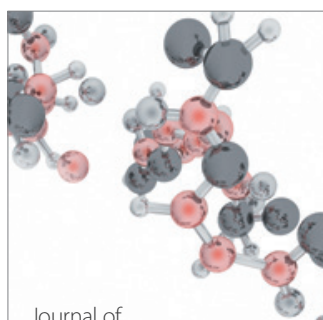

Analytical Methods

in Chemistry

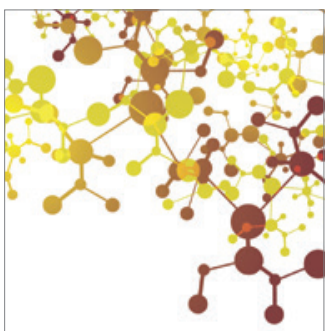

Journal of

Applied Chemistry

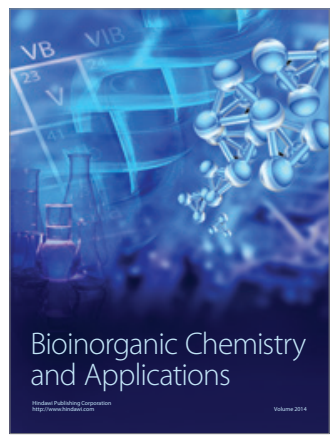

Inorganic Chemistry
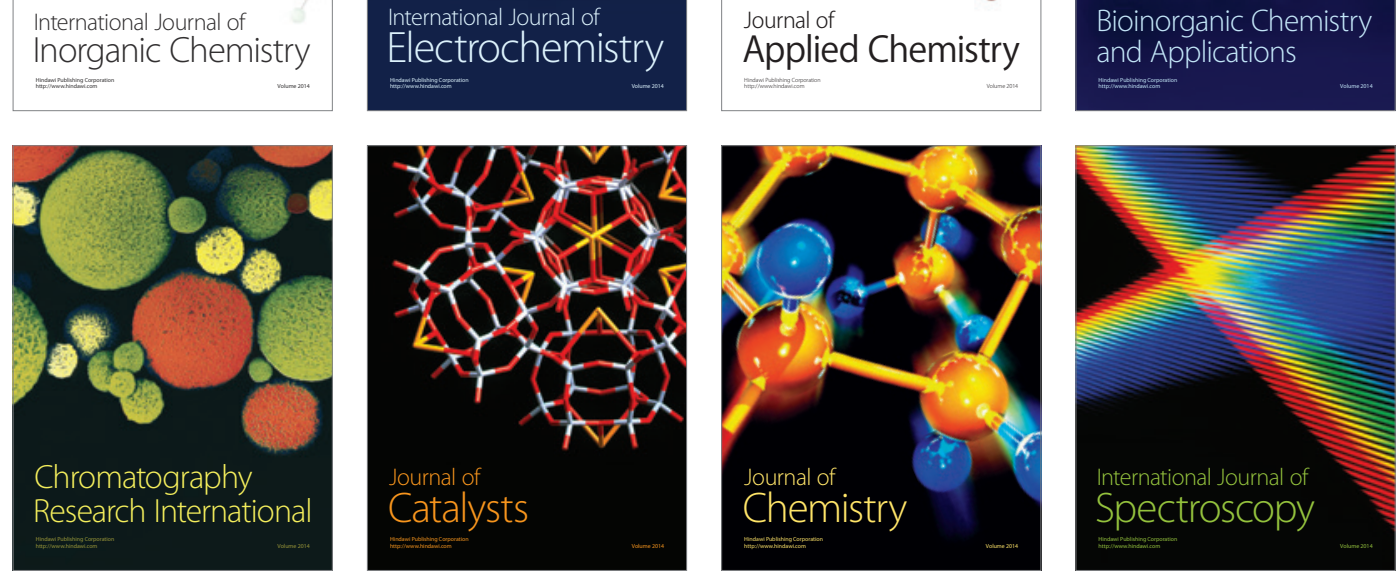